\title{
Balancing Health and High Performance: Carlos Jiménez, MD, MSpMed Deputy Director, Sports Medicine Institute, Havana
}

\section{By Kathleen Vickery, MA}

The Sports Medicine Institute (abbreviated in Spanish as IMD) in Havana is a hub of energy concentrated on the health and training of the nation's top athletes. While fencing teams, wrestlers and judokas work out in one building, a multidisciplinary staff keeps office hours next door for athletes, retired athletes, their families and others referred to their services. And not just "office" hours: everyone at the IMD, including doctors, seem to put physical activity into practice, moving in and out of hallways, up and down stairs, and along pathways between buildings and outdoor training areas, as if keeping pace with the young athletes in the lead.

It is a dynamic, high-performance environment, reflecting the close interaction among all players on the medicalathletic teams, working towards the combined goals of gold-medal achievement and lifetime health and fitness.

Overseeing the IMD's day-to-day activities nationwide since 1999 is Deputy Director Dr Carlos Jiménez Amaro, former judoka and, more recently, Chief Medical Officer of the Cuban delegation to the 2008 Beijing Olympics. Dr Jiménez spoke with MEDICC Review about the evolution of sports medicine in Cuba and its role in the country's health system.

MEDICC Review: For a small country of limited resources, Cuba has an impressive international competition record: in medal-count history, the country ranks first in the Central American and Caribbean Games, second in the Pan American Games, and its overall Olympic ranking stands at 23 out of 128 countries. In fact, Cuba is the only Latin American or Caribbean country in the Olympic top 30 . What role does sports medicine play in preparing athletes and protecting their health at these high performance levels?

Carlos Jiménez: First of all, sports medicine has been an integral part of athletic training in Cuba since the National Sports, Physical Education and Recreation Institute (INDER) was founded in 1961. Before then, few Cubans had the opportunity to participate in a range of organized competitive sports, and sports medicine as a medical speciality did not exist. As INDER began to build a comprehensive physical education and athletic program, and make it accessible to the whole population, sports medicine developed along with it.

Initially a Sports Medicine Department was created in Havana, staffed by a few doctors whose main role was taking care of injured athletes. Since this was clearly insufficient, in 1964, a group of five physicians went abroad for specialized training and returned two years later to set up a post-graduate program in sports

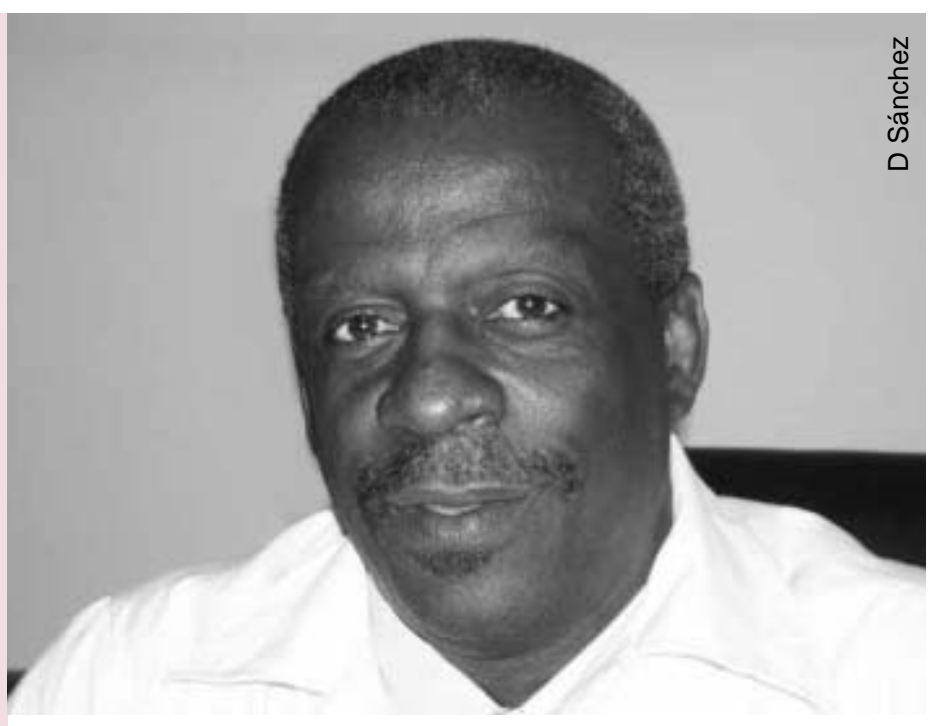

medicine, under the auspices of the Ministry of Public Health. In 1970, the first graduates of that program introduced "medical management of athletic conditioning" into the training program for all athletes in the high-performance pyramid. The program continued to grow, and in 1976, the Sports Medicine Institute was founded.

MEDICC Review: What exactly is the high-performance pyramid?

Carlos Jiménez: The high-performance pyramid comprises some 26,000 athletes nationwide, children and adults, including 54 national sports teams. It is quite simple: school-age children who want to play sports begin through programs in elementary school. If they show aptitude and want to become athletes, they may be selected to go to a school that combines regular classes with sports training appropriate for their age and ability. Those children make up the broad base of the pyramid, but they are still considered high-performance athletes because they work toward top performance for their age, body type and sport.

In the middle of the pyramid are older youth, most of whom combine high school and pre-university studies with athletic training and competition. They make up the talent pool from which the country's top-performing athletes are ultimately drawn-the adults on the national and Olympic teams.

MEDICC Review: What does medical management of athletic conditioning involve, and how is it applied at each level of the pyramid?

Carlos Jiménez: Medical management includes a physical, psychological and nutritional evaluation of each athlete, individually 
and with respect to the requirements of each sport. It is basically a holistic approach that seeks to balance the athlete's physical and mental well-being with performance demands.

For each sport, at each level, doctors work closely with psychologists, physical therapists and nutritionists to prepare and monitor each athlete's training load and diet, taking into account their sex, biological age, chronological age, anthropometric profile and other factors in relation to the performance demands of their sport.

Each national team-the ones at the top of the pyramid-has a full-time sports doctor, psychologist and physical therapist-the 'medical triad,' as we call it-who, together with the coach, make up the technical team in charge of coordinating the health status, training load and performance of these athletes, individually and as a team.

\section{MEDICC Review: What about injury prevention? Top athletes are constantly pushed to exceed their physical limits. How do you balance that against the risk of injury?}

Carlos Jiménez: Medical management of athletes as they move up through the pyramid means that by the time they are selected for the top teams, we know them very well. We have helped guide their development and conditioning, and we know their health history. The triad uses this information to develop an individualized injury prevention plan for each athlete.

In addition, training programs are evaluated and modified regularly, depending on athletes' response to the training load. Conditioning for each sport is planned using a periodized training protocol that varies the intensity and volume of specific exercises throughout a season, or macrocycle. For example, the Olympic foil fencing team's 2008-2009 training cycle comprises 32 weeks beginning in October with a preparatory period that lasts through March, followed by a competition period in April and a brief transition period in May. Within each of these periods, training is organized in mesocycles of varying duration and with specific performance goals: general conditioning and sport-specific training during the preparatory period; performance achieved and stabilized during competition; and relief during the transition period. Within each mesocycle, the intensity and volume of exercise is determined in weekly, or minicycle, segments, and progressively increased up to and including championship games in the spring.

The medical triad sees the team every day and evaluates athletes at specific points in the cycle when the training load is increased. If the athlete passes the physiological and psychological exams, and shows no signs of excess stress or overuse, then the doctor gives the okay for her or him to advance and increase the training load. If, however, his or her performance is less than optimum for any reason, the doctor may recommend continuing at the same pace. Or, if there is any sign of injury or serious difficulty, the doctor may recommend that training be modified until recovery. At that point, the team doctor oversees treatment and rehabilitation, which may include referrals to other specialists within IMD or at one of the hospitals we work with.

The medical triad applies this same method to detraining when an athlete retires from high-performance competition, but with a plan that gradually reduces, rather than increases, the volume and intensity of conditioning.

\section{Chronology of Sports Medicine in Cuba}

Year

before 1961 No national sports program or sports medicine specialty in Cuba

National Sports, Physical Education and Recreation

1961 Institute (INDER) created, including a Sports Medicine Department

1964 Group of five Cuban doctors go abroad for specialized training in sports medicine

1966 Sports medicine speciality established by Ministry of Public Health

1967 Sports medicine residency program initiated

Sports medicine residency program graduates first

1970 specialists who initiate medical management of athletic conditioning with all athletes in the high-performance pyramid

1976 National Sports Medicine Institute (Instituto Cubano de Medicina del Deporte-IMD) founded

1992 IMD initiates Master's program in Medical Management of Athletic Conditioning

2001 IMD opens Anti-Doping Laboratory

IMD's Anti-Doping Laboratory accredited by the Inter-

2003 national Olympic Committee and the World Anti-Doping Agency

\section{MEDICC Review: Tell us more about detraining.}

Carlos Jiménez: I'd say this is one of the outstanding features of sports medicine in Cuba: "forgotten" is not a word we use with "athlete." The IMD provides lifetime health care for all former highperformance athletes and their families here in Havana and at IMD centers in all 14 provinces and the Isle of Youth-wherever ex-athletes live.

Detraining helps retiring athletes make a healthy physical and psychological adjustment to a lifestyle no longer geared to intensive physical conditioning and competition. The technical team prepares a plan for tapering an athlete's training load-still considering all the physical, psychological and nutritional aspects I mentioned before-and a plan for continuing physical exercise, adequate diet and so on that will help the person stay healthy and in shape. With detraining, ex-athletes are better prepared to prevent chronic conditions such as obesity, diabetes and hypertension, as well as musculoskeletal problems and illnesses related to smoking and alcoholism.

When athletes leave high-performance competition, they confront a whole new set of expectations in their families and communities. In a way, their friends and families also have to adjust to their becoming ex-athletes. And that shift in expectations often creates new stresses in their relationships and daily life. Ex-athletes are also more exposed to unhealthy habits in social situations than they were before. So, family and community support is very important. The detraining program actively involves athletes' families, and sometimes their neighbors and community organizations, to create a supportive environment. At the same time, it encourages athletes to set an example and promote exercise and fitness to those around them.

Finally, as I mentioned before, all high-performance athletes and their families continue to receive health care through the IMD. 
MEDICC Review: What kind of sports medicine services are provided for athletes' families and the community at large?

Carlos Jiménez: In addition to sports medicine specialists, the IMD has a full range of medical personnel on staff, including dentists, psychologists, and physical therapists. Together they provide comprehensive services here in Havana and at the IMD Center in each province and on the Isle of Youth. Our first priority is the medical management of athletic conditioning and detraining for all the athletes in the pyramid, as I mentioned before, but athletes' families can also come to us for any health problem. Although they all receive free health care through the regular public health system, sometimes they prefer to come to us, or find it more convenient, because we already have a close relationship through our work with the athletes themselves.

All IMD centers are open to the community, but most IMD doctors provide services to the general population in rotations through community-based polyclinics in each municipality. In both situations, they see mostly patients referred by family doctors or other specialists for a variety of health problems that may be alleviated, at least in part, through an exercise program. These include, of course, hypertension and cardiovascular disease, musculoskeletal problems, and obesity.

MEDICC Review: Does the IMD have any programs aimed at preventing those health problems at the community level?

Carlos Jiménez: Yes. Every IMD Center has a health promotion service that applies some of the same elements of medical management of athletic conditioning to the non-athlete population referred to us. We apply many of the same techniques for evaluating physical development and functionality, cardiovascular functioning, and other factors that we use with athletes, taking into consideration the patient's original diagnosis. We then prescribe therapy, which usually includes an exercise program, sometimes combined with nutrition and psychological consultations, plus physical therapy. And we organize classes and exercise programs that help patients "fill their prescriptions" but that are open to anyone.

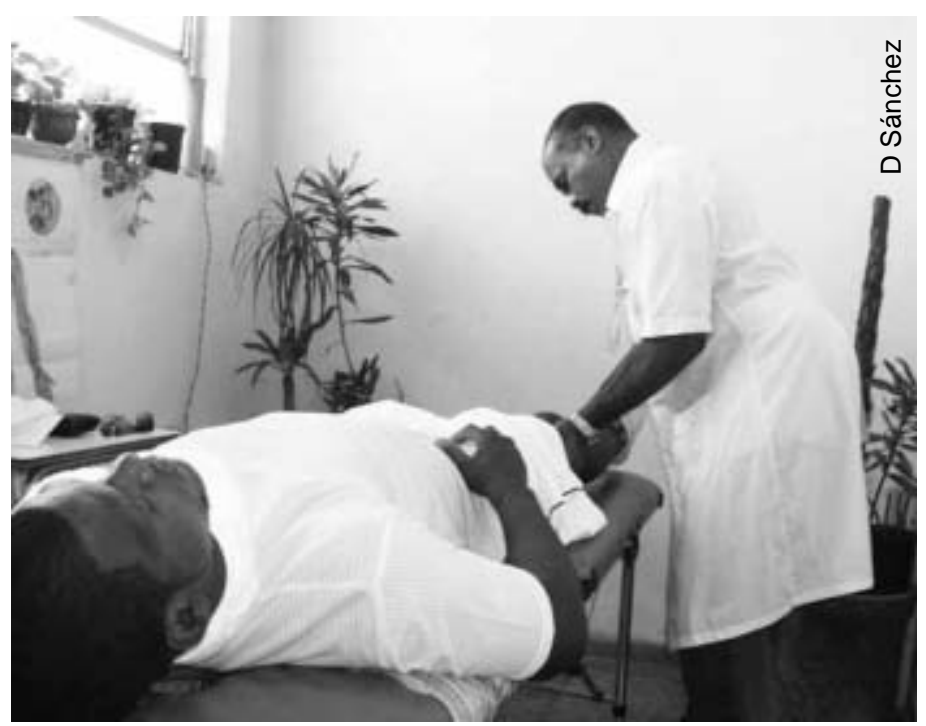

Physical therapy session at the Sports Medicine Institute in Havana.
I should add that the IMD is also a teaching and research institution. The medical staff includes full and adjunct professors in sports medicine and other specialties who conduct the sports medicine residency program and a Master's program in medical management of athletic conditioning. The IMD also maintains close research and academic ties with major Cuban hospitals, particularly the Frank País Orthopedic Teaching Hospital in Havana and its Sports Traumatology department.

IMD faculty direct the research projects required of our residents and master's candidates, and one of our main areas of investigation focuses on the preventive and therapeutic applications of physical exercise for improving quality of life, especially for people with noncommunicable chronic diseases.

These are the most prevalent health problems in Cuba today, even among ex-athletes. In 2008, just in Havana, 447 former athletes participated in detraining but less than half-151-were considered in good health. Of the majority, 134 had hypertension, 35 were diabetic, 38 had heart disease, 12 were asthmatic, 19 had musculoskeletal problems, and 58 were classified as obese. So, we are studying ways to improve both the impact of detraining on these indicators as well as the outcomes of our health promotion program with non-athletes.

There is no question that more needs to be done to educate people about the benefits of exercise. We would like to be able to expand the IMD's health promotion program and provide permanent sports medicine services in at least one polyclinic in every municipality, but the residency program is small, and we don't yet have that capacity. From a sports medicine perspective, promoting exercise and physical activity is important, but what is most effective-particularly with older adults and people with chronic conditions-is medical management of an exercise program suited to each person's characteristics. The same principles that apply to conditioning for high-performance sports can apply to conditioning for quality of life. - - p 